\title{
Mass distribution in the most X-ray-luminous galaxy cluster RX J1347.5-1145 studied with XMM-Newton
}

\author{
M. Gitti ${ }^{1}$, R. Piffaretti ${ }^{2}$, and S. Schindler ${ }^{3}$ \\ 1 INAF - Osservatorio Astronomico di Bologna, via Ranzani 1, 40127 Bologna, Italy \\ e-mail: myriam.gitti@oabo.inaf.it \\ 2 SISSA/ISAS, via Beirut 4, 34014 Trieste, Italy \\ 3 Institut für Astro- und Teilchen Physik, Leopold-Franzens Universität Innsbruck, Technikerstraße 25, 6020 Innsbruck, Austria
}

Received 1 April 2007 / Accepted 18 June 2007

\begin{abstract}
Context. We report on the analysis of XMM-Newton observations of RX J1347.5-1145 $(z=0.451)$, the most X-ray-luminous galaxy cluster.

Aims. We present a detailed total and gas mass determination up to large distances $(\sim 1.7 \mathrm{Mpc})$, study the scaling properties of the cluster, and explore the role of AGN heating in the cluster cool core.

Methods. By means of spatially resolved spectroscopy we derive density, temperature, entropy, and cooling time profiles of the intracluster medium. We compute the total mass profile of the cluster in the assumption of hydrostatic equilibrium.

Results. If the disturbed south-east region of the cluster is excluded from the analysis, our results on shape, normalization, scaling properties of density, temperature, entropy, and cooling time profiles are fully consistent with those of relaxed, cool core clusters. We compare our total and gas mass estimates with previous X-ray, lensing, dynamical, and SZ studies. We find good agreement with other X-ray results, dynamical mass measurements, weak lensing masses and SZ results. We confirm a discrepancy of a factor $\sim 2$ between strong lensing and X-ray mass determinations and find a gross mismatch between our total mass estimate and the mass reconstructed through the combination of both strong and weak lensing. We explore the effervescent heating scenario in the core of RX J1347.5-1145 and find support to the picture that AGN outflows and heat conduction are able to quench radiative cooling.
\end{abstract}

Key words. galaxies: clusters: individual: RX J1347.5-1145 - X-rays: galaxies: clusters - galaxies: intergalactic medium galaxies: cooling flows - cosmology: dark matter - cosmology: observations

\section{Introduction}

X-ray observations of the diffuse Intra-Cluster Medium (ICM) in clusters of galaxies are a particularly rich source of information for understanding the formation of large scale structure and the physics of clusters. As they are the last manifestation of hierarchical clustering, whose history depends strongly on cosmology, galaxy clusters are key objects for cosmological studies (see Voit 2005, for a review). Since the evolution of the ICM is mainly driven by the gravity of the underlying dark matter halo, clusters are expected to show similar properties when rescaled with respect to their total mass and formation epoch. However, deviations from self-similarity are expected under the effect of more complex physical processes, beyond gravitational dynamics only, which affect the thermodynamical properties of the diffuse ICM (e.g. Evrard \& Henry 1991; Bryan \& Norman 1998; Borgani et al. 2002, and references therein). It is therefore essential to investigate whether galaxy clusters obey the expected scaling relations, which are the foundation to use these virialized objects as cosmological probes. The first important step in this context is to find a proxy for an accurate determination of the cluster mass.

The galaxy cluster RX J1347.5-1145 $(z=0.451)$ is an exceptional object in many aspects. It is the most X-ray-luminous cluster known to date $\left(L_{X}=6 \times 10^{45} \mathrm{erg} \mathrm{s}^{-1}\right.$ in the [2-10] keV energy range) with a very peaked surface brightness profile and hosts a strong cooling flow in its center with nominal mass accretion rate of $\sim 1900 M_{\odot} \mathrm{yr}^{-1}$ (Gitti \& Schindler 2004). The cluster is dominated by two cD galaxies which are separated by about $\sim 18^{\prime \prime}$ along the east-west direction, the X-ray emission being centered on the western one. Although this is unusual for strong cooling flow clusters, the optical spectrum of the western Brightest Cluster Galaxy (BCG) indicates that it hosts an active galactic nucleus (AGN), with typical emission lines of giant ellipticals at the center of cooling flow clusters (Cohen \& Kneib 2002). More striking is a recent discovery made with Chandra (Allen et al. 2002b) and XMM-Newton (Gitti \& Schindler 2004) of a region with hot, bright X-ray emission located at $\sim 20$ arcsec from the central emission peak in south-east direction. Millimeter observations previously detected a very deep SZ decrement in the south-east region of the cluster (Komatsu et al. 2001; Pointecouteau et al. 2001). These results were interpreted as indications of a subcluster merger in an otherwise relaxed, massive cool core cluster, pointing to a complex dynamical evolution of the system. Furthermore, RX J1347.5-1145 is a powerful gravitational lens and mass reconstructions based on weak and strong lensing analyses have been performed (Schindler et al. 1995; Fischer \& Tyson 1997; Sahu et al. 1998; Bradač et al. 2005b).

With a detailed study of the properties of the ICM in this cluster it is thus possible to address many key issues on both dynamical and non-gravitational processes in galaxy clusters. A great advantage of observing RX J1347.5-1145 with XMM-Newton is that important quantities derived for the undisturbed cluster (i.e., with the south-east quadrant excluded) such as the azimuthally averaged ICM density and temperature 
profiles can be computed up to a large distance from the center $(\sim 1730 \mathrm{kpc})$. The measurement of cluster temperature gradients at large distances is also crucial for determining the total gravitational masses and in turn the gas mass fraction of clusters. A precise determination of the total mass at large radii allows an estimate of the virial radius of the object without much extrapolation of the universal NFW dark matter profile (Navarro et al. 1996). The virial radius can then be used to study the scalings of the temperature and entropy profiles and a fair comparison between predictions of numerical simulations and observations can be performed. Currently, the two most promising techniques for obtaining accurate determinations of cluster masses are $\mathrm{X}$-ray observations, by deprojection of X-ray surface brightness combined with spectroscopic determination of the cluster temperature, and gravitational lensing, through either strong lensing features or statistical distortions of background objects (weak lensing). The mass estimates inferred with these two methods can be quite inconsistent, particularly in the case of strong lensing (e.g. Wu et al. 1998, and references therein). In contrast to the X-ray technique, the gravitational lensing method is essentially free of assumptions on the nature and the dynamical state of the gravitating material. In particular, the X-ray method can be affected strongly during mergers (Schindler 1996) and in the inner cluster region where a strong interaction between the central AGN and the ICM is present (e.g., Bîrzan et al. 2004), as in these cases deviations from the assumptions of hydrostatic equilibrium and spherical symmetry are expected. Since both the total mass profile derived from X-rays and the total mass distribution derived from gravitational lensing are available for RX J1347.5-1145, a comparison between them is possible thus providing important insights on this issue. Furthermore, the presence of gas with short cooling time in the cluster core offers the opportunity to explore gas heating processes such as AGN heating, which have become increasingly popular since the failure of standard cooling flows models.

In this paper, by starting from the results of morphological (Sect. 3) and spectral (Sect. 4) analyses of XMM-Newton observations of RX J1347.5-1145 (Sect. 2), we present a detailed study of the cluster mass distribution (Sect. 6), and discuss its comparison with the mass profile derived from previous studies (Sect. 7). We also study the scaling properties of the cluster (Sects. 5 and 6) and explore the role of AGN heating in the cluster cool core in the context of the effervescent heating scenario (Sect. 8). RX J1347.5-1145 (hereafter RX J1347) is at a redshift of 0.451 . With a Hubble constant of $H_{0}=70 \mathrm{~km} \mathrm{~s}^{-1} \mathrm{Mpc}^{-1}$, and $\Omega_{\mathrm{M}}=1-\Omega_{\Lambda}=0.3$, the luminosity distance is $2506 \mathrm{Mpc}$ and the angular scale is $5.77 \mathrm{kpc}$ per arcsec.

\section{Observation and data preparation}

RX J1347 was observed by XMM-Newton in July 2002 during rev. 484 with the MOS and pn detectors in Full Frame Mode with THIN filter, for an exposure time of $37.8 \mathrm{ks}$ for MOS and $33.2 \mathrm{ks}$ for pn. We use the SASv6.0.0 processing tasks emchain and epchain to generate calibrated event files from raw data. Throughout this analysis single pixel events for the pn data (PATTERN 0) are selected, while for the MOS data sets the PATTERNs 0-12 are used. The removal of bright pixels and hot columns is done in a conservative way applying the expression $(F L A G==0)$. To reject the soft proton flares we accumulate the light curve in the [10-12] keV band for MOS and [12-14] keV band for pn, where the emission is dominated by the particleinduced background, and exclude all the intervals of exposure time having a count rate higher than a certain threshold value (the chosen threshold values are $0.15 \mathrm{cps}$ for MOS and $0.22 \mathrm{cps}$ for $\mathrm{pn})$. The remaining exposure times after cleaning are $32.2 \mathrm{ks}$ for MOS1, $32.5 \mathrm{ks}$ for MOS2 and $27.9 \mathrm{ks}$ for pn. Starting from the output of the SAS detection source task, we make a visual selection on a wide energy band MOS \& pn image of point sources in the field of view (hereafter FOV). Events from these regions are excluded directly from each event list.

The background estimates are obtained using a blank-sky observation consisting of several high-latitude pointings with sources removed (Lumb et al. 2002). The blank-sky background events are selected using the same selection criteria (such as PATTERN, FLAG, etc.), intensity filter (for flare rejection) and point source removal used for the observation events; this yields final exposure times for the blank fields of $365 \mathrm{ks}$ for MOS1, $350 \mathrm{ks}$ for MOS2 and $294 \mathrm{ks}$ for pn. Since the cosmic ray induced background might slightly change with time, we compute the ratio of the total count rates in the high energy band ([10-12] keV for MOS and [12-14] keV for pn). The obtained normalization factors $(0.992,1.059,1.273$ for MOS1, MOS2 and pn, respectively) are then used to renormalize the blank field data. Furthermore, the blank-sky background files are recast in order to have the same sky coordinates as RX J1347. The background subtraction (for spectra and surface brightness profiles) is performed as described in full detail in Arnaud et al. (2002). This procedure consists of two steps. In a first step, for each product extracted from the observation event list, an equivalent product is extracted from the corresponding blank-field file and then subtracted from it. This allows us to remove the particle background. However, if the background in the observation region is different from the average background in blank field data, this step could leave a residual background component. The residual background component is estimated by using blank field subtracted data in a region free of cluster emission and then subtracted in a second step from each MOS and pn product.

The source and background events are corrected for vignetting using the weighted method described in Arnaud et al. (2001), the weight coefficients being tabulated in the event list with the SAS task evigweight. This allows us to use the on-axis response matrices and effective areas. Unless otherwise stated, the reported errors are at $90 \%$ confidence level.

\section{Surface brightness profile}

Previous Chandra and XMM-Newton observations of RX J1347 revealed the presence of a hot and bright X-ray subclump visible to the south-east (SE) of the main X-ray surface brightness peak (Allen et al. 2002b; Gitti \& Schindler 2004). On the other hand, the data excluding the SE quadrant (hereafter "undisturbed cluster") show a regular morphology, indicating a relaxed state. We are interested in determining the characteristic properties of the cluster in order to perform studies of mass profiles and scaling relations as it is usually done for relaxed clusters. The disturbed SE quadrant is thus masked in the following morphological analysis.

We compute a background-subtracted, vignetting-corrected, radial surface brightness profile in the [0.3-2] keV energy band for each camera separately. For the pn data, we generate a list of out-of-time events ${ }^{1}$ (hereafter OoT) to be treated as an additional background component. The effect of OoT in the current observing mode (Full Frame) is 6.3\%. The OoT list is processed

\footnotetext{
1 Out-of-time events are caused by photons which arrive while the CCD is being read out, and are visible in an uncorrected image as a bright streak smeared out in RAWY.
} 


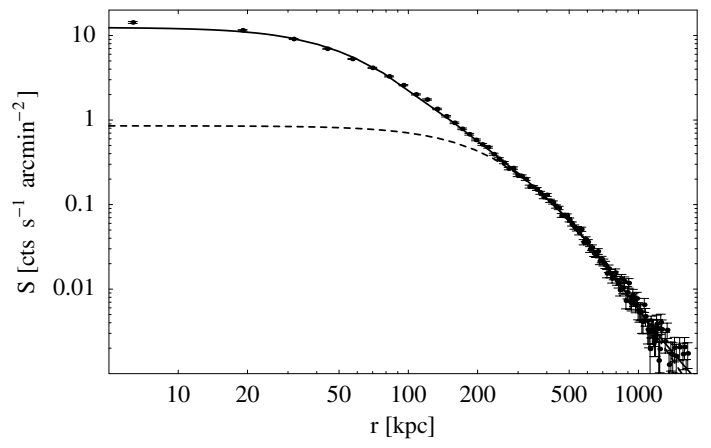

Fig. 1. Background subtracted, azimuthally-averaged radial surface brightness profile in the [0.3-2] $\mathrm{keV}$ range of the data excluding the SE quadrant (undisturbed cluster). The best fit $\beta$-model fitted over the $\sim 350-1730 \mathrm{kpc}$ region is over-plotted as a dashed line (model SO in Table 1). When extrapolated to the center, this model shows a strong deficit as compared to the observed surface brightness. The solid line shows the best fit double $\beta$-model fitted over the whole region (model DD in Table 1).

in a similar way as done for the pn observation event file. The profiles for the three detectors are then added into a single profile, binned such that at least a signal-to-noise ratio of 3 is reached. The cluster emission is detected up to $R_{\text {out }}=1.73 \mathrm{Mpc}$ ( $\sim 5 \mathrm{arcmin})$. The surface brightness profile of the undisturbed cluster, shown in Fig. 1, is fitted in the CIAO tool Sherpa with various parametric models, which are convolved with the XMM point spread function (PSF). The overall PSF is obtained by adding the PSF of each camera (Ghizzardi 2001), estimated at an energy of $1.5 \mathrm{keV}$ and weighted by the respective cluster count rate in the [0.3-2] keV energy band. A single $\beta$-model (Cavaliere \& Fusco-Femiano 1976):

$S(r)=S_{0}\left(1+\frac{r^{2}}{r_{\mathrm{c}}^{2}}\right)^{-3 \beta+0.5}$

is not a good description of the entire profile (model SG in Table 1) and a fit to the outer regions (350 kpc $\lesssim r \lesssim 1730 \mathrm{kpc}$ ) shows a strong excess in the center as compared to the model (see Fig. 1). The centrally peaked emission is a strong indication of a cooling flow in this cluster. We find that for $350 \mathrm{kpc} \lesssim r \lesssim$ $1730 \mathrm{kpc}$ the data can be described by a $\beta$-model with a core radius $r_{\mathrm{c}}=307 \pm 9 \mathrm{kpc}$ and a slope parameter $\beta=0.86 \pm 0.02$ ( $3 \sigma$ confidence level). The single $\beta$-model functional form is a convenient representation of the gas density profile in the outer regions, which is used as a tracer for the potential. The parameters of this best fit are thus used in the following to estimate the cluster gas and total mass profiles in the region where the single $\beta$-model holds (see Sect. 6).

We also consider a double isothermal $\beta$-model in the form:

$S(r)=\sum_{i} S_{0, i}\left(1+\frac{r^{2}}{r_{\mathrm{c}, i}^{2}}\right)^{-3 \beta_{i}+0.5}$

where $i=1,2$, and find that it can account for the entire profile (see Fig. 1). The best fit parameters are $r_{\mathrm{c}, 1}=39 \pm 1 \mathrm{kpc}, \beta_{1}=$ $0.62 \pm 0.01, r_{\mathrm{c}, 2}=386 \pm 17 \mathrm{kpc}, \beta_{2}=1.01 \pm 0.05$. By assuming a common $\beta$ value we find: $r_{\mathrm{c}, 1}=241 \pm 7 \mathrm{kpc}, r_{\mathrm{c}, 2}=47 \pm 2 \mathrm{kpc}$, $\beta=0.76 \pm 0.01$ (see Table 1$)$.

\section{Spectral analysis}

Throughout the analysis, a single spectrum is extracted for each region of interest and is then regrouped to reach a significance level of at least 25 counts in each bin. The data are modeled using the XSPEC code, version 11.3.0 (Arnaud 1996). Unless otherwise stated,the relative normalizations of the MOS and pn spectra are left free when fitted simultaneously. We use the following response matrices: m1_439_im_pal1_v1.2.rmf (MOS1), m2_439_im_pall_v1.2.rmf (MOS2), epn_ff20_sY9.rmf (pn).

\subsection{Global spectrum}

For each instrument, a global spectrum is extracted from all events lying within 5 arcmin to the cluster emission peak. We test in detail the consistency between the three camera by fitting separately these spectra with a mekal model (with the redshift fixed at $z=0.451$ ) absorbed by a column density included in the tbabs model (fixed at the nominal galactic value $N_{\mathrm{H}}=$ $4.85 \times 10^{20} \mathrm{~cm}^{-2}$, Dickey \& Lockman 1990). Fitting the data from all instruments above $0.3 \mathrm{keV}$ leads to inconsistent values for the temperature derived with the MOS and pn cameras: $k T=12.2_{-0.6}^{+0.7} \mathrm{keV}(\mathrm{MOS} 1), 10.4_{-0.5}^{+0.5} \mathrm{keV}(\mathrm{MOS} 2), 9.3_{-0.3}^{+0.3} \mathrm{keV}$ (pn). We then perform a systematic study of the effect of imposing various high and low-energy cutoffs, for each instrument. Good agreement between the three cameras is found in the $[0.8-10.0] \mathrm{keV}$ energy range $\left(k T=11.2_{-0.6}^{+0.7} \mathrm{keV}\right.$ for MOS1, $10.0_{-0.5}^{+0.6}$ for MOS2, $10.2_{-0.4}^{+0.4}$ for $\left.\mathrm{pn}\right)$. We therefore perform the spectral analysis in this energy range. The combined MOS+pn global temperature, in $\mathrm{keV}$, and metallicity, as a fraction of the solar value (Anders \& Grevesse 1989) derived from the best fit $\left(\chi^{2} /\right.$ d.o.f. $\left.=2717 / 1697\right)$ are respectively: $k T=10.4_{-0.3}^{+0.3} \mathrm{keV}$, $Z=0.25_{-0.03}^{+0.03} Z_{\odot}$. The unabsorbed luminosities in this model (estimated from the average of the fluxes measured by the three cameras after fixing $\left.N_{\mathrm{H}}=0\right)$ in the X-ray $([2.0-10.0] \mathrm{keV})$ and bolometric band are respectively: $L_{X}=6.2 \pm 0.2 \times 10^{45} \mathrm{erg} \mathrm{s}^{-1}$, $L_{\mathrm{bol}}=13.5 \pm 0.4 \times 10^{45} \mathrm{erg} \mathrm{s}^{-1}$, where the errors are given as half the difference between the maximum and the minimum value.

\subsection{Spatially resolved spectra}

As done for the morphological analysis, for the spectral analysis we separate the SE quadrant containing the X-ray subclump from the rest of the cluster. The data of the undisturbed cluster are divided into the following annular regions: $0-30^{\prime \prime}, 30^{\prime \prime}-1^{\prime}$, $1^{\prime}-1.5,1$ '.5-2., 2.'-3', 3.-5'. The spectra are modeled using a simple, single-temperature model (mekal plasma emission code in XSPEC) with the absorbing column density fixed at the nominal Galactic value. The free parameters in this model are the temperature $k T$, metallicity $Z$ (measured relative to the solar values, with the various elements assumed to be present in their solar ratios, Anders \& Grevesse 1989) and normalization (emission measure). The best-fitting parameter values and $90 \%$ confidence levels derived from the fits to the annular spectra are summarized in Table 2.

\subsection{Deprojection analysis}

Because of projection effects, the spectral properties at any point in the cluster are the emission-weighted superposition of radiation originating at all points along the line of sight through the cluster. To correct for this effect, we perform a deprojection analysis by adopting the XSPEC projct model. Under the assumption of ellipsoidal (in our specific case, spherical) shells of emission, this model calculates the geometric weighting factor 
Table 1. Results from fitting the surface brightness profile of the undisturbed cluster in different radial intervals $\left[R_{\text {in }}-R_{\text {out }}\right]$. The single and double $\beta$-models used for the fitting are given by Eqs. (1) and (2), respectively. They are indicated with: SG (Single $\beta$-model, fitted in the Global radial range), SO (Single $\beta$-model, fitted in the Outer region), DD (Double $\beta$-model, with Different $\beta$ values), DE (Double $\beta$-model, with Equal $\beta$ values). The quoted errors are at $3 \sigma$ confidence level.

\begin{tabular}{|c|c|c|c|c|c|c|c|}
\hline Model & $\begin{array}{r}R_{\mathrm{i}} \\
(\operatorname{arcmin})\end{array}$ & $\begin{array}{l}R_{\text {out }} \\
\quad(\mathrm{kpc})\end{array}$ & $\begin{array}{c}S_{0, i} \\
\left(\mathrm{cts} / \mathrm{s} / \operatorname{arcmin}^{2}\right)\end{array}$ & $\beta_{i}$ & $\begin{array}{r}r_{\mathrm{c}, i} \\
(\operatorname{arcmin})\end{array}$ & $(\mathrm{kpc})$ & $\chi^{2} /$ d.o.f. $\left(\chi_{\text {red }}^{2}\right)$ \\
\hline SG: single $\beta$ & $0.0-5.0$ & $0-1731$ & $14.42_{-0.50}^{+0.50}$ & $0.590_{-0.005}^{+0.005}$ & $0.1492_{-0.0030}^{+0.0029}$ & $52_{-1}^{+1}$ & $1620 / 129(12.56)$ \\
\hline SO: single $\beta$ & $1.0-5.0$ & $346-1731$ & $0.891_{-0.075}^{+0.075}$ & $0.861_{-0.020}^{+0.022}$ & $0.8876_{-0.0265}^{+0.0253}$ & $307_{-9}^{+9}$ & $109 / 87(1.25)$ \\
\hline $\begin{array}{l}\text { DD: double } \beta \\
\text { with } \beta_{1} \neq \beta_{2}\end{array}$ & $0.0-5.0$ & $0-1731$ & $\begin{array}{c}18.94_{-0.86}^{+0.86} \\
0.42_{-0.04}^{+0.04}\end{array}$ & $\begin{array}{l}0.616_{-0.008}^{+0.009} \\
1.010_{-0.043}^{+0.051}\end{array}$ & $\begin{array}{c}0.1138_{-0.0033}^{+0.0032} \\
1.1145_{-0.0506}^{+0.0483}\end{array}$ & $\begin{array}{c}40_{-1}^{+1} \\
386_{-18}^{+17}\end{array}$ & 258/111 (2.32) \\
\hline $\begin{array}{l}\text { DE: double } \beta \\
\text { with } \beta_{1}=\beta_{2}\end{array}$ & $0.0-5.0$ & $0-1731$ & $\begin{array}{c}18.12_{-1.00}^{+1.00} \\
0.96_{-0.06}^{+0.06}\end{array}$ & $0.761_{-0.011}^{+0.012}$ & $\begin{array}{l}0.1360_{-0.0050}^{+0.0048} \\
0.6968_{-0.0211}^{+0.0205}\end{array}$ & $\begin{array}{c}47_{-2}^{+2} \\
241_{-7}^{+7}\end{array}$ & $289 / 112(2.58)$ \\
\hline
\end{tabular}

Table 2. Results of the spectral fitting in concentric annular regions in the [0.8-10.0] keV energy range obtained by fixing the absorbing column density to the Galactic value $\left(N_{\mathrm{H}}=4.85 \times 10^{20} \mathrm{~cm}^{-2}\right)$. The temperature (in keV) and metallicity (in fraction of the solar value, Anders \& Grevesse 1989) are left as free parameters. The data of the SE quadrant are excluded (undisturbed cluster).

\begin{tabular}{ccccc}
\hline \hline $\begin{array}{c}\text { Radius } \\
(\mathrm{kpc})\end{array}$ & $\begin{array}{c}\text { source counts } \\
(\mathrm{MOS}+\mathrm{pn})\end{array}$ & $\begin{array}{c}k T \\
(\mathrm{keV})\end{array}$ & $\begin{array}{c}Z \\
\left(Z_{\odot}\right)\end{array}$ & $\chi^{2} /$ d.o.f. \\
\hline $0-173$ & 46719 & $9.3_{-0.3}^{+0.3}$ & $0.31_{-0.05}^{+0.05}$ & $914 / 964$ \\
$173-346$ & 18377 & $12.5_{-0.9}^{+1.1}$ & $0.16_{-0.01}^{+0.01}$ & $573 / 546$ \\
$346-519$ & 8733 & $11.8_{-1.2}^{+1.5}$ & $0.22_{-0.15}^{+0.14}$ & $288 / 295$ \\
$519-692$ & 4331 & $9.4_{-1.3}^{+1.7}$ & $0.13_{-0.13}^{+0.18}$ & $201 / 178$ \\
$692-1038$ & 4092 & $9.8_{-1.7}^{+2.5}$ & $0.18_{-0.18}^{+0.25}$ & $315 / 229$ \\
$1038-1731$ & 2742 & $7.3_{-2.3}^{+4.2}$ & $0.40_{-0.40}^{+0.64}$ & $572 / 383$ \\
\hline
\end{tabular}

according to which the emission is redistributed amongst the projected annuli.

The deprojection analysis is performed by fitting simultaneously the spectra of the three cameras. The results are reported in Table 3 . We also calculate the electron density $n_{\mathrm{e}}$ from the estimate of the Emission Integral $E I=\int n_{\mathrm{e}} n_{\mathrm{p}} \mathrm{d} V$ given by the mekal normalization: $10^{-14} E I /\left(4 \pi\left[D_{\mathrm{A}}(1+z)\right]^{2}\right)$. We assume $n_{\mathrm{e}}=1.2023 n_{\mathrm{p}}$ in the ionized intra-cluster plasma.

\section{Radial profiles}

\subsection{Temperature}

The deprojected temperature profile derived in Sect. 4.3 is shown in Fig. 2, where we also show the projected profile for comparison. As expected, the deprojected central temperature is lower than the projected one, since in the projected fits the spectrum of the central annulus is contaminated by hotter emission along the line of sight. We also note that the projected temperature profile measured by Chandra (Allen et al. 2002b) is systematically slightly higher than that measured by XMM-Newton, although the general trend observed by the two satellites is consistent (Gitti \& Schindler 2004).

The temperature profile of RX J1347 exhibits the shape characteristic for cool core clusters: the temperature declines from the maximum cluster temperature at a break radius $r_{\mathrm{br}}$ moving outwards and drops towards the cluster center. If $r_{\mathrm{br}}$ is simply defined as the distance from the cluster center where the

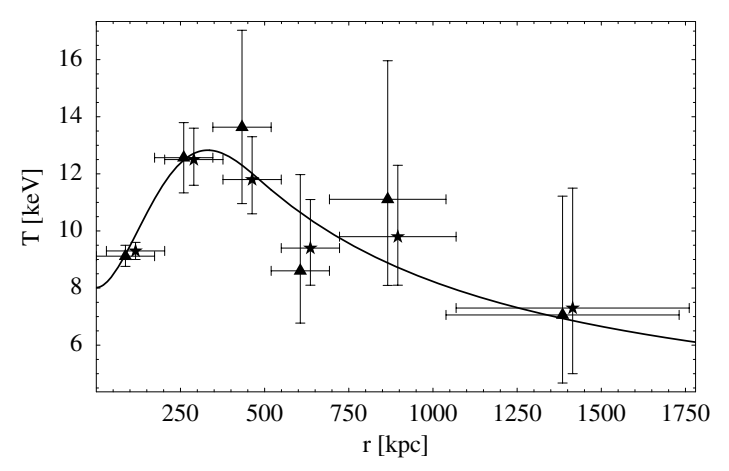

Fig. 2. Deprojected (triangles) and projected (stars) X-ray gas temperature profiles measured in the $[0.8-10.0] \mathrm{keV}$ energy range. The data points of the projected profile are slightly shifted to the right to improve the clarity of the plot. The solid line shows the best fit function used in the total gravitational mass estimation presented in Sect. 6.1 below.

temperature is maximal, then $r_{\mathrm{br}}=433 \pm 87 \mathrm{kpc}$ for the deprojected profile and $r_{\mathrm{br}}=260 \pm 87 \mathrm{kpc}$ for the projected profile, respectively. This distance corresponds to $\sim 0.1-0.2 r_{\text {vir }}$ (see Sect. 6.2), in agreement with works on the scaling properties of large samples of clusters of galaxies (Markevitch et al. 1998; De Grandi \& Molendi 2002; Piffaretti et al. 2005; Vikhlinin et al. 2005; Pratt et al. 2007). The temperature decrease observed in the outer regions $\left(\sim 40 \%\right.$ from $r_{\text {br }}$ to $0.5 r_{\text {vir }}$ ) is also consistent with the findings of these studies. The temperature derived from the deprojected spectral analysis drops from the peak value of $13.6 \mathrm{keV}$ to the central minimum value of $9.1 \mathrm{keV}$. This is fully consistent with the typical $30 \%$ drop seen in temperature profiles of cool core clusters (e.g. see Kaastra et al. 2004).

\subsection{Cooling time}

The cluster RX J1347 is known to host a cool core (Schindler et al. 1997; Allen et al. 2002b; Gitti \& Schindler 2004). The centrally peaked surface brightness profile and the central temperature drop discussed in Sects. 3 and 5.1, respectively, are indeed signatures of the presence of a central region where the plasma cooling time is short. In the following we compute the cooling time profile and the cooling radius of the cluster.

The cooling time is calculated as the characteristic time that it takes a plasma to cool isobarically through an increment of temperature $\delta T$ :

$t_{\text {cool }}=\frac{5}{2} \frac{k \delta T}{n_{\mathrm{e}} \Lambda(T)}$ 
Table 3. Results of the deprojection analysis on annular MOS + pn spectra using the XSPEC projct model. The column density is fixed to the Galactic value and the normalizations are in units of $10^{-14} n_{\mathrm{e}} n_{\mathrm{p}} V / 4 \pi\left[D_{\mathrm{A}}(1+z)\right]^{2}$. The fit gives $\chi^{2} /$ d.o.f. $=3007 / 2557$. The data of the SE quadrant are excluded (undisturbed cluster).

\begin{tabular}{lcccc}
\hline \hline $\begin{array}{l}\text { Radius } \\
(\mathrm{kpc})\end{array}$ & $\begin{array}{c}k T \\
(\mathrm{keV})\end{array}$ & $\begin{array}{c}Z \\
\left(Z_{\odot}\right)\end{array}$ & $\begin{array}{c}\text { norm } \\
\left(\times 10^{-3}\right)\end{array}$ & $\begin{array}{c}n_{\mathrm{e}} \\
\left(\times 10^{-3} \mathrm{~cm}^{-3}\right)\end{array}$ \\
\hline $0-173$ & $9.1_{-0.4}^{+0.4}$ & $0.32_{-0.05}^{+0.05}$ & $6.02_{-0.08}^{+0.08}$ & $23.22_{-0.16}^{+0.16}$ \\
$173-346$ & $12.6_{-1.2}^{+1.2}$ & $0.16_{-0.15}^{+0.12}$ & $2.73_{-0.07}^{+0.09}$ & $5.91_{-0.08}^{+0.09}$ \\
$346-519$ & $13.6_{-2.7}^{+3.4}$ & $0.22_{-0.21}^{+0.30}$ & $1.51_{-0.09}^{+0.07}$ & $2.66_{-0.08}^{+0.06}$ \\
$519-692$ & $8.6_{-1.8}^{+3.4}$ & $0.18_{-0.18}^{+0.25}$ & $0.88_{-0.06}^{+0.06}$ & $1.46_{-0.05}^{+0.05}$ \\
$692-1038$ & $11.1_{-3.0}^{+4.9}$ & $0.08_{-0.08}^{+0.39}$ & $0.81_{-0.07}^{+0.04}$ & $0.69_{-0.03}^{+0.02}$ \\
$1038-1731$ & $7.1_{-2.4}^{+4.2}$ & $0.39_{-0.39}^{+0.59}$ & $0.54_{-0.07}^{+0.08}$ & $0.25_{-0.02}^{+0.02}$ \\
\hline
\end{tabular}

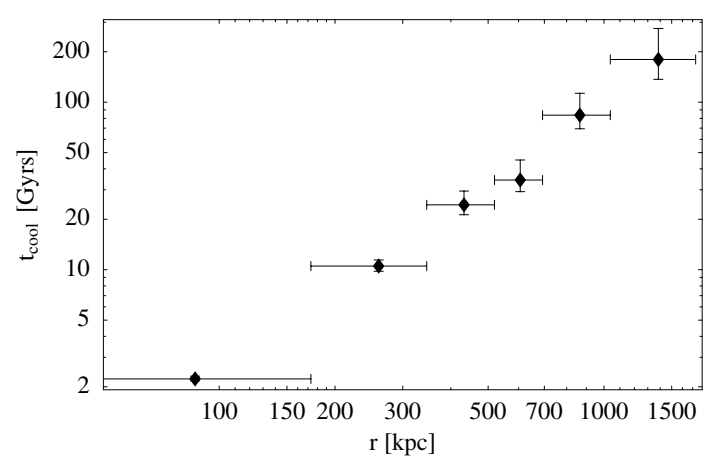

Fig. 3. Cooling time as a function of radius.

where $\Lambda(T)$ is the total emissivity of the plasma (the cooling function) and $k$ is Boltzmann's constant. Utilizing the deprojected temperature profile and the density profile from Sect. 4.3, we can calculate the cooling time as a function of radius, which is shown in Fig. 3. The cooling time shows a power law behavior as a function of radius. We find $t_{\text {cool }} \propto r^{1.46 \pm 0.01}$ when all 6 radial bins are used in the fit and $t_{\text {cool }} \propto r^{1.72 \pm 0.21}$ if only the 4 radial bins beyond $0.2 r_{500} \approx 280 \mathrm{kpc}$ are considered (see Sect. 6.2 below for the definition and computation of $r_{500}$ ). The latter value agrees with recent results from the analysis in the same radial range of a sample of luminous clusters at $z=0.2$ (Zhang et al. 2007). Following Bîrzan et al. (2004), we define the cooling radius as the radius within which the gas has a cooling time less than $7.7 \times 10^{9} \mathrm{yr}$, the look-back time to $z=1$ for our adopted cosmology. With this definition, we find $r_{\text {cool }} \sim 210 \pm 10 \mathrm{kpc}$ which corresponds to the central 36 arcsec.

In the following analysis it is important to correct for the effects of the central cooling flow when measuring the characteristic temperature of the undisturbed cluster. The average emission-weighted cluster temperature is calculated by fitting with a mekal model the spectrum extracted up to the outer radius detected by our X-ray observation ( 5 arcmin), after excising the cooling region (central 35 arcsec) and the SE quadrant. We find a value $\left\langle T_{\mathrm{X}}\right\rangle=10.1 \pm 0.7 \mathrm{keV}$.

\subsection{Entropy}

The gas entropy in groups and clusters of galaxies has recently received particular attention since it resulted to be a very useful quantity to probe the thermodynamic history of the hot gas in these systems. The entropy is usually defined as $S=k T / n_{\mathrm{e}}^{2 / 3}$, where $T$ and $n_{\mathrm{e}}$ are the deprojected electron temperature and density, respectively.

In cooling core clusters the radial entropy profiles are expected to increase monotonically moving outwards, and to show no isentropic cores (e.g., McCarthy et al. 2004). This behavior is indeed observed in nearby cooling core clusters (Piffaretti et al. 2005; Pratt et al. 2006). Entropy profiles are in general well described by a power law. The value of the power law index scatters around unity, depending on the cluster or cluster sample used to derive it: for example, Ettori et al. (2002a) found 0.97 from Chandra data of A1795, Pratt \& Arnaud (2005) derived a slope of $0.94 \pm 0.14$ from scalings of the entropy profiles of 5 clusters observed with $X M M-N e w t o n$, Piffaretti et al. (2005) found $0.95 \pm 0.02$ using scaled profiles of 13 cool core clusters observed with XMM-Newton, and Pratt et al. (2006) derived a slope of $1.08 \pm 0.04$ (extending the sample studied in Pratt \& Arnaud (2005) to 10 objects).

In Fig. 4 we show the gas entropy profile of RX J1347 computed from the deprojected temperature and electron density derived in Sect. 4.3. We fit the profile with a line in log$\log$ space (with errors in both coordinates) and find: $\log [S]=$ $(1.053 \pm 0.005) \times \log [r]+(0.011 \pm 0.010)$ (entropy in $\mathrm{keV} \mathrm{cm}{ }^{2}$ and radius in $\mathrm{kpc}$ ), which is consistent with previous results. Donahue et al. (2006) recently found that the entropy profiles they derived from Chandra observations of 9 cool core clusters are better fitted by a power law plus a constant entropy pedestal of $\approx 10 \mathrm{keV} \mathrm{cm}^{2}$ than by a pure power law. We performed similar fits and find an entropy pedestal consistent with zero. However, we notice that this result might be due to the lack of adequate spatial resolution of the entropy profile in the central region.

Recent results suggest that the entropy scales with the temperature as $S \propto\left\langle T_{\mathrm{X}}\right\rangle^{0.65}$, the so-called "entropy ramp", instead of the self-similar scaling $S \propto\left\langle T_{\mathrm{X}}\right\rangle$ (Ponman et al. 2003; Pratt \& Arnaud 2005; Piffaretti et al. 2005; Pratt et al. 2006). Here $\left\langle T_{\mathrm{X}}\right\rangle$ is the mean cluster/group temperature corrected for the cool core effect and $S$ is the entropy measured at some fraction of he virial radius (usually $0.1 \times r_{200}$, see Sect. 6.2 below for the definition and computation of $r_{200}$ ). In order to verify if the entropy measured in RX J1347 follows this relation, we therefore adopt the scaling $S \propto h^{-4 / 3}(z)\left(\left\langle T_{\mathrm{X}}\right\rangle / 10 \mathrm{keV}\right)^{0.65}$, with a mean temperature for RX J1347 equal to $10.1 \pm 0.7 \mathrm{keV}$ (see Sect. 5.2). Here $h^{2}(z)=\Omega_{\mathrm{m}}(1+z)^{3}+\Omega_{\Lambda}$ and the factor $h^{-4 / 3}$ comes from the scaling of the density. At $0.1 \times r_{200}$ the scaled entropy is equal to $382 \pm 32,349 \pm 54$, and $437 \pm 51 \mathrm{keV} \mathrm{cm}^{2}$ for $r_{200}$ computed from the total mass profiles derived from model SO, DDg1, and NFW, respectively (see Sect. 6.1 below for the different models used in the total mass determination from the X-ray data). If instead the value $r_{200}^{\mathrm{Sim}}$ is used (i.e., we adopt the size-temperature relation calibrated through numerical simulations, see Sect. 6.2 below), the normalization is $567 \pm 70 \mathrm{keV} \mathrm{cm}^{2}$. The errors on these normalizations also take into account the uncertainty in the estimate of $r_{200}$. The normalization derived by adopting the sizetemperature relation is in good agreement with the entropy normalization of the $S\left(0.1 \times r_{200}\right)-\left\langle T_{\mathrm{X}}\right\rangle$ relation at $\left\langle T_{\mathrm{X}}\right\rangle=10 \mathrm{keV}$ by Ponman et al. (2003, see their Fig. 4). The values computed using $r_{200}$ derived from the total mass profiles are smaller, but still consistent within the uncertainties, than the values found by Ponman et al. (2003).

\section{Mass determination}

In Gitti \& Schindler (2004) we presented the total mass profile estimated from the single $\beta$-model. Here we perform a detailed study of the radial profiles of total gravitational mass and gas 


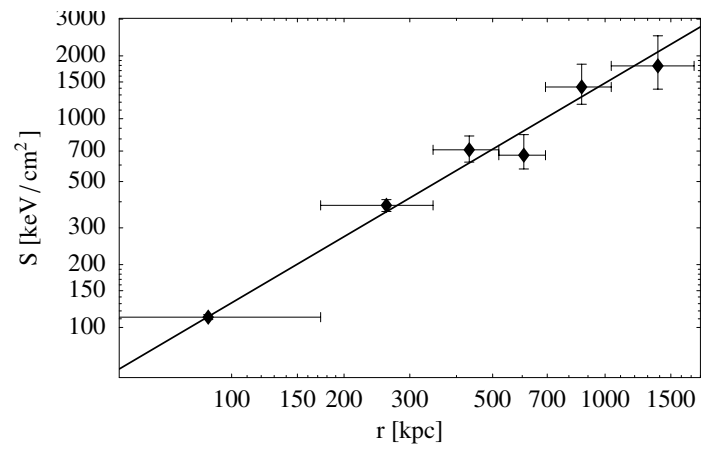

Fig. 4. Entropy as a function of radius and the best fit power law $\log [S]=(1.053 \pm 0.005) \times \log [r]+(0.011 \pm 0.010)$ (entropy in $\mathrm{keV} \mathrm{cm}{ }^{2}$ and radius in $\mathrm{kpc})$.

mass reconstructed by using different methods. The new values do not change the main conclusions in Gitti \& Schindler (2004) but are more accurate. In this section we also present the computation of the characteristic radii $r_{\Delta}$ quoted above.

\subsection{Total gravitational mass}

The analysis to estimate the total gravitational mass of RX J1347 is not limited to only one specific method, but is instead carried out by adopting different approaches. This enables us to investigate the effects introduced by different fitting functions for the gas density and temperature, and different methods to derive the total mass from the observed gas distribution.

The total gravitating mass distribution is calculated under the usual assumptions of hydrostatic equilibrium and spherical symmetry by using

$M_{\text {tot }}(<r)=-\frac{k T(r) r}{G \mu m_{\mathrm{p}}}\left[\frac{\mathrm{d} \ln \rho_{\mathrm{g}}(r)}{\mathrm{d} \ln r}+\frac{\mathrm{d} \ln T_{\mathrm{g}}(r)}{\mathrm{d} \ln r}\right]$

where $G$ and $m_{\mathrm{p}}$ are the gravitational constant and proton mass and $\mu=0.62$. A welcome property of Eq. (4) is that the total gravitational mass within a sphere of radius $r$ is determined from the gas density $\rho_{\mathrm{g}}$ and temperature $T_{\mathrm{g}}$ measured at the clustercentric distance $r$. This implies that when the gas density and temperature are well modeled only in the radial range $R_{\text {in }}-R_{\text {out }}$ but not within $R_{\text {in }}$, the mass determination is still reliable in the range $R_{\text {in }}-R_{\text {out }}$. As shown in Sect. 3, a single $\beta$-model provides a good fit to the surface brightness profile in the radial range $350 \mathrm{kpc} \lesssim r \lesssim 1730 \mathrm{kpc}$ (model SO in Table 1$)$. In this case the deprojected gas density profile is easily computed and the total cluster mass is independent of the gas density central value. Since beyond $350 \mathrm{kpc}$ the temperature profile is declining, it can be well modeled through the polytropic relation $T \propto \rho_{\mathrm{g}}^{\gamma-1}$, with $1 \leq \gamma \leq 5 / 3$. The polytropic fit to the deprojected temperature profiles gives in this case $\gamma=1.23 \pm 0.02$ ( $1 \sigma$ error on one parameter). The total mass profile computed using this model is discussed below together with the results from the more sophisticated double $\beta$-model.

In order to obtain a total mass estimate for the whole observed radial range we use the double $\beta$-model fits discussed in Sect. 3 (model DD and DE in Table 1). The gas density is computed from the double $\beta$-model surface brightness fits using the formulas derived in Xue \& Wu (2000): we assume that each component corresponds to a gas phase, invert Eq. (2) and compute the electron number densities for the two components $n_{\mathrm{e}, \mathrm{i}}(r)$ and the total electron number density, $n_{\mathrm{e}}(r)$ using:

$$
\begin{aligned}
& n_{\mathrm{e}}(r)=\sum_{i} n_{\mathrm{e}, i}(r)=\left[n_{\mathrm{e}}(0) \sum_{i} \tilde{n}_{\mathrm{e}, i}(r)\right]^{1 / 2}, \\
& n_{\mathrm{e}, i}(r)=\left[\frac{n_{\mathrm{e}}(0)}{n_{\mathrm{e}}(r)}\right] \tilde{n}_{\mathrm{e}, i}(r), \\
& \tilde{n}_{\mathrm{e}, i}(r)=n_{\mathrm{e}, i}(0)\left(1+\frac{r^{2}}{r_{\mathrm{c}, i}^{2}}\right)^{-3 \beta_{i}},
\end{aligned}
$$

where $i=1,2$ and $n_{\mathrm{e}}(0)$ is the central, total electron density. The central number densities for the two components are given by

$$
n_{\mathrm{e}, i}^{2}(0)=\left[\frac{4 \pi^{1 / 2}}{\alpha\left(T_{i}\right) g_{i} \mu_{\mathrm{e}}}\right]\left[\frac{\Gamma\left(3 \beta_{i}\right)}{\Gamma\left(3 \beta_{i}-1 / 2\right)}\right]\left(\frac{S_{0, i}}{r_{\mathrm{c}, i}}\right) A_{i j}
$$

in which

$$
\frac{1}{A_{i j}}=1+\left(\frac{g_{i}}{g_{j}}\right)\left(\frac{r_{\mathrm{c}, i} S_{0, j}}{r_{\mathrm{c}, j} S_{0, i}}\right)\left(\frac{T_{i}}{T_{j}}\right)^{1 / 2}\left[\frac{\Gamma\left(3 \beta_{j}\right) \Gamma\left(3 \beta_{i}-1 / 2\right)}{\Gamma\left(3 \beta_{i}\right) \Gamma\left(3 \beta_{j}-1 / 2\right)}\right],
$$

where $j=1,2$ and $j \neq i$. Here $g_{i}$ is the Gaunt factor for the component $i$ and $\alpha\left(T_{i}\right)$ is the emissivity due to thermal bremsstrahlung. The Gaunt factors are computed using the results of Sutherland (1998). Note that in the derivation of the equations given above it is assumed that each component has a constant electron temperature $T_{i}$ throughout the cluster. As shown in Sect. 5 the gas is not isothermal hence this assumption is not strictly valid. Nevertheless the temperature dependence of the above equation is fairly weak and we set $T_{1 \text { or } 2}=$ $T_{\max }=13.6 \mathrm{keV}$ (the maximum of the temperature profile) and $=T_{2 \text { or } 1}=T_{\min }=7.1 \mathrm{keV}$ (the minimum of the temperature profile) to quantify the maximum variation of the total mass estimate with temperature. Using the above equations and Eq. (4) we compute the mass profile for 4 cases: DDg1 (model DD and $\left.T_{1}=T_{\max }, T_{2}=T_{\min }\right), \operatorname{DDg} 2\left(\operatorname{model} \mathrm{DD}\right.$ and $T_{1}=T_{\min }, T_{2}=$ $T_{\max }$ ), DEg1 (model DE and $T_{1}=T_{\max }, T_{2}=T_{\min }$ ), and DEg2 (model DE and $T_{1}=T_{\min }, T_{2}=T_{\max }$ ). While the assumption of isothermality is justified in the evaluation of the densitydependent term of Eq. (4) from the observed surface brightness profile, the radial dependence of the gas temperature must be carefully modeled, since the total gravitational mass varies strongly with temperature. The temperature profile in the whole observed range is clearly not well described by a polytropic relation and it is not possible to model it using a single analytical function due to the central temperature drop. We therefore model the profile using two functions joined smoothly at a cut radius $R_{\text {cut }}$, i.e. we take care that the temperature profile and its gradient are continuous across $R_{\text {cut }}$. Since the polytropic relation provides a good description in the outer region, we adopt $T \propto \rho_{\mathrm{g}}^{\gamma-1}$ as fitting function for $r \geq R_{\text {cut }}$, with $\rho$ computed from the double $\beta$-model fits. The values obtained for the parameter $\gamma$ are very similar to those obtained when using the single $\beta$ model. Within $R_{\text {cut }}$ we choose to fit the temperature profile using a 5 th order polynomial with zero derivative at the center. If the latter condition is not satisfied the derived total mass density is found to be negative in the cluster core. We vary $R_{\text {cut }}$ and find that $R_{\text {cut }}=520 \mathrm{kpc}$ provides the best model. The resulting best fit function is shown in Fig. 2. The total mass profiles computed from the surface brightness fits presented in the following are computed using this temperature profile modeling and will be 
indicated by the name of the model used to describe the surface brightness (see Table 1).

The relative difference between the mass profiles for model DDg1 and DDg2 (DEg1 and DEg2) is less than 4\% (6\%) in the whole observed radial range $(0-1731 \mathrm{kpc})$. Models DDg1 and DDg2, and DEg1 and DEg2 give nearly identical results for $r>500 \mathrm{kpc}$. The largest difference is found between models $\mathrm{DDg} 1$ and DEg2, but it is less that $15 \%$ in the whole radial range and less than $5 \%$ for $r>250$. These small differences show that the temperature does not significantly affect the gas density determination for this massive and hot cluster, and that models DE an DD provide the same mass estimate for the whole radial range of interest. Given these results and the fact that model DD gives a smaller $\chi_{\text {red }}^{2}$ than model DE for the surface brightness modeling, we will discuss, in the following, only the mass profile derived using model DDg1. We compare the mass profiles derived from the double $\beta$-model with the one from the single $\beta$ model in the radial range $350-1731 \mathrm{kpc}$. In this range the relative difference of the mass profiles is at most $13 \%$ (close to the innermost and outermost radii), but smaller than $10 \%$ in the range $380-1500 \mathrm{kpc}$ for the four double $\beta$-models we derived. Hence, the double $\beta$-model provides estimates in good agreement with the single $\beta$-model, and is of course preferred since it allows us to estimate the mass in the whole observed radial range, i.e. $0-1731 \mathrm{kpc}$. The mass profiles from the double $\beta$-model (model DDg1) and the single $\beta$-model (model SO) are plotted in Fig. 5. Errors on the total gravitational masses are computed by propagating the $1 \sigma$ errors on the surface brightness and temperature profiles best fit parameters, and are of the order of $10 \%$ and $20 \%$ for the values derived from the single and double $\beta$ model, respectively. The profile derived using the single $\beta$-model is shown only in the region where it is valid, i.e. for $r>350$. The mild depression visible around $\sim 250 \mathrm{kpc}$ in the mass profile derived from the double $\beta$-model is due to the shape of the temperature profile in the inner region.

The cluster gravitational mass can also be computed by making direct use of the gas temperature and gas density profiles derived from the deprojection analysis presented in Sect. 4.3. We invert the equation of hydrostatic equilibrium (Eq. (4)) and, using the three-dimensional gas density, we select the dark matter mass model that reproduces better the deprojected temperature profile. In the minimization the $1 \sigma$ errors on one single parameter from the spectral fits are used. For dark matter mass model, we consider the integrated NFW (Navarro et al. 1996) dark matter profile:

$M_{\mathrm{DM}}(<r)=4 \pi r_{\mathrm{s}}^{3} \rho_{\mathrm{c}, \mathrm{z}} \frac{200}{3} \frac{c^{3}\left(\ln \left(1+r / r_{\mathrm{s}}\right)-\frac{r / r_{\mathrm{s}}}{\left(1+r / r_{\mathrm{s}}\right)}\right)}{\ln (1+c)-c /(1+c)}$,

where $\rho_{\mathrm{c}, z}=\left(3 H_{z}^{2}\right) /(8 \pi G)$ is the critical density at the cluster's redshift. The scale radius $r_{\mathrm{s}}$ and the concentration parameter $c$ are the free parameters. The total gravitational mass within a sphere of radius $r$ is given by gas plus dark matter mass and therefore $M_{\mathrm{tot}}(<r)=M_{\mathrm{gas}}(<r)+M_{\mathrm{DM}}(<r)$ in Eq. (4). Nevertheless, in most of the work $M_{\mathrm{tot}}(<r)=M_{\mathrm{DM}}(<r)$ is used, i.e. the NFW profile is used to fit dark matter plus gas mass. We also computed the total mass profile by taking into account the gas mass, i.e. by adding the cumulative gas mass profile to the best-fitting NFW profile, and found little difference between the two profiles. The best-fit parameters are $r_{\mathrm{s}}=722 \pm 112 \mathrm{kpc}$ and $c=3.20 \pm 0.30$ (errors are rms of the $1 \sigma$ joint confidence limits), with $\chi_{\min }^{2}=6.7$ for 4 degrees of freedom. Our best-fit NFW profile is shown in Fig. 5. From the set of $\left(c, r_{\mathrm{s}}\right)$ parameters acceptable at $1 \sigma$ we compute, for each radius, the maximum

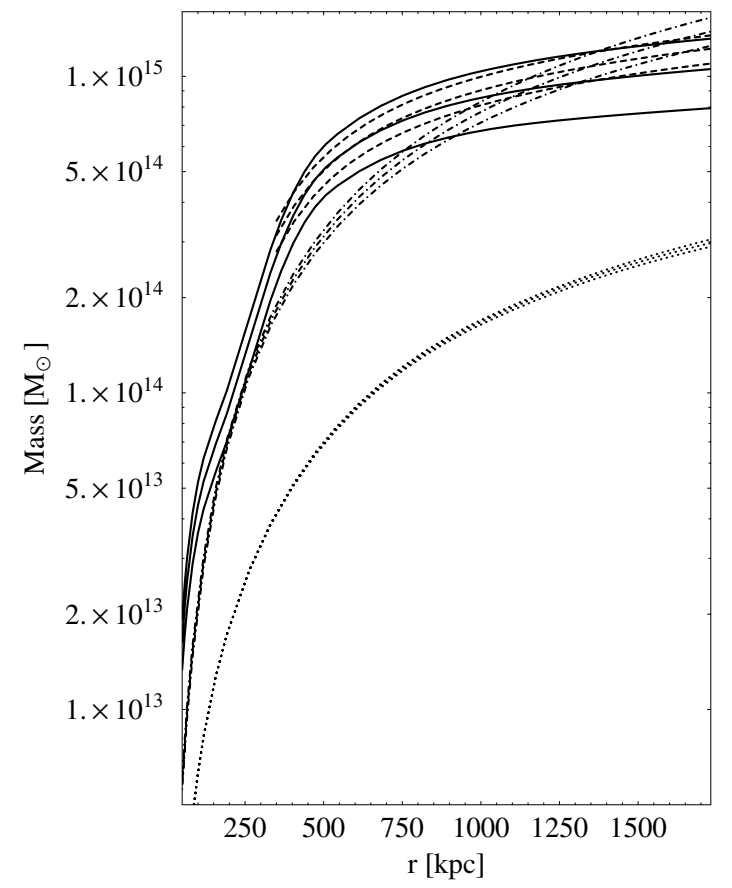

Fig. 5. Integrated three dimensional total mass profiles, with errors, derived from the double $\beta$-model (model DDg1, solid), single $\beta$-model (model SO, dashed), and the NFW model (dot-dashed). The dotted line shows the cumulative gas mass profile. See text for details.

and minimum value of the total mass and hence its upper and lower errors. These are of the order of $10 \%$. From a visual inspection of Fig. 5 one can note that the NFW mass profile is lower than the double $\beta$ estimate for $r<1150 \mathrm{kpc}$ and higher at larger radii. The discrepancy within $1150 \mathrm{kpc}$ is due to the fact that our best-fit NFW profile tends to underestimate the temperature in this range. The relative difference between the NFW and the double $\beta$ mass profiles is $-38 \%$ (underestimate) at $r=500$ but decreasing towards the center, and increases almost linearly to $30 \%$ (overestimate) at $r=1731 \mathrm{kpc}$. The fairly low concentration parameter $c$, compared to the predictions of numerical simulations (e.g., Macciò et al. 2006), and the goodness of our NFW fit might indicate that our temperature profile is not enough spatially resolved in the central region of the cluster for this kind of mass determination method. While the mass determination from the double $\beta$-model may therefore be preferred, we present values also from the NFW fitting for completeness.

\subsection{Virial radius and scaling relations}

In this section we determine the characteristic radii $r_{\Delta}$ used in Sects. 5 and 5.3. For the various mass profiles we compute $r_{\Delta}$, the radius within which the mean interior density is $\Delta$ times the critical value, by using

$\Delta=\frac{3 M_{\mathrm{tot}}\left(<r_{\Delta}\right)}{4 \pi \rho_{\mathrm{c}, z} r_{\Delta}^{3}}$

For the cosmology adopted here the virial radius is given by $r_{\text {vir }}=r_{\tilde{\Delta}}$, with $\tilde{\Delta}=178+82 x-39 x^{2}$ and where $x=\Omega(z)-1$ and $\Omega(z)=0.3(1+z)^{3} /\left(0.3(1+z)^{3}+0.7\right)$ (Bryan \& Norman 1998). Thus for RX J1347 $\tilde{\Delta}=135$. We also compute $M_{\text {tot }}\left(<r_{\Delta}\right)$ and $M_{\text {gas }}\left(<r_{\Delta}\right)$ for various overdensities: $\Delta=2500,1000,500,200$. The results obtained from the overdensity profiles calculated from the double $\beta$-model (DDg1) and NFW fit are reported in Table 4. 
Table 4. Characteristic radii $r_{\Delta}$, total mass $M_{\mathrm{tot}}$ and gas mass $M_{\mathrm{gas}}$ for various overdensities $\Delta$ derived from the double $\beta$-model (DDg1) and NFW fits ( $1 \sigma$ errors in parentheses). The masses are estimated within $r_{\Delta}$. As discussed in Sect. 6.1, results from the double $\beta$-model are generally more reliable.

\begin{tabular}{ccccccc}
\hline \hline$\Delta$ & $\begin{array}{c}r_{\Delta, \mathrm{DDg} 1} \\
(\mathrm{kpc})\end{array}$ & $\begin{array}{c}M_{\text {tot,DDg1 }} \\
\left(10^{14} M_{\odot}\right)\end{array}$ & $\begin{array}{c}M_{\text {gas,DDg1 }} \\
\left(10^{14} M_{\odot}\right)\end{array}$ & $\begin{array}{c}r_{\Delta, \mathrm{NFW}} \\
(\mathrm{kpc})\end{array}$ & $\begin{array}{c}M_{\text {tot,NFW }} \\
\left(10^{14} M_{\odot}\right)\end{array}$ & $\left.\begin{array}{c}M_{\text {gas,NFW }} \\
\left(10^{14}\right.\end{array} M_{\odot}\right)$ \\
\hline 200 & $1957.2(183.2)$ & $11.00(2.78)$ & $3.34(0.11)$ & $2286.7(110.8)$ & $17.86(2.07)$ & $3.82(0.18)$ \\
500 & $1387.2(123.9)$ & $9.77(2.30)$ & $2.39(0.05)$ & $1479.2(71.23)$ & $11.85(1.13)$ & $2.55(0.06)$ \\
1000 & $1063.3(91.00)$ & $8.80(1.91)$ & $1.79(0.03)$ & $1029.3(43.08)$ & $7.99(0.62)$ & $1.73(0.03)$ \\
2500 & $729.3(63.2)$ & $7.10(1.40)$ & $1.15(0.02)$ & $608.1(19.8)$ & $4.12(0.22)$ & $0.91(0.01)$ \\
\hline
\end{tabular}

The size-temperature relation $r_{\Delta} \propto \sqrt{\left\langle T_{\mathrm{X}}\right\rangle}$ predicted by selfsimilarity allows an estimate of $r_{\Delta}$ from the mean cluster temperature alone, provided that its normalization is known from numerical simulations. We compute the normalization for the cosmology adopted here by interpolating the values given in Evrard et al. (1996). For the mean cluster temperature $\left\langle T_{\mathrm{X}}\right\rangle=$ $10.1 \pm 0.7 \mathrm{keV}$ we derive the characteristic radii $r_{\Delta}^{\text {Sim }}$, finding $r_{2500}^{\mathrm{Sim}}=886 \pm 30 \mathrm{kpc}$ and $r_{\mathrm{vir}}^{\mathrm{Sim}}=3197 \pm 107 \mathrm{kpc}$. From our $\mathrm{X}$-ray analysis we find $r_{2500}=(734 \pm 34,729 \pm 63,608 \pm 20) \mathrm{kpc}$ and $r_{\text {vir }}=(2378 \pm 76,2241 \pm 189,2639 \pm 108) \mathrm{kpc}$ when using in Eq. (11) the mass profile derived from model (SO, DDg1, NFW), respectively. These values are consistent with the sizetemperature relation derived from observations of nearby relaxed clusters (Arnaud et al. 2005). By comparing the above values we note that the estimates from the X-ray analysis are systematically lower than the ones predicted from the size-temperature relation calibrated by means of numerical simulations. It is not surprising that we find a smaller discrepancy for $r_{2500}$ than $r_{\mathrm{vir}}$, as its determination does not require extrapolation of the observed mass profile. This is in agreement with results for other individual clusters (e.g., Gitti et al. 2007a) and studies of cluster samples (Sanderson et al. 2003; Piffaretti et al. 2005). The largest discrepancy is found for $r_{\mathrm{vir}}$ and in poor, cool clusters. In these systems the impact of additional, non-gravitational heating is most pronounced, as the extra energy required to account for their observed properties is comparable to their thermal energy (Ponman et al. 1996; Tozzi \& Norman 2001). The observed discrepancy is also related to the cluster total mass determination. In this context it is interesting to note that recent results from numerical simulations indicate that the total mass of simulated clusters estimated through the X-ray approach is lower that the true one due to gas bulk motions (i.e. deviation from the hydrostatic equilibrium) and the complex thermal structure of the gas (Rasia et al. 2006; Nagai et al. 2007).

A self-similar scaling relation between $M_{\text {tot }}$ and $\left\langle T_{\mathrm{X}}\right\rangle$ at a given overdensity is predicted in the form $M_{\mathrm{tot}} \propto\left\langle T_{\mathrm{X}}\right\rangle^{3 / 2}$. Various observational studies have found different and sometime conflicting results regarding the slope and normalization of the $M-T$ relation (e.g., Allen et al. 2001; Finoguenov et al. 2001; Ettori et al. 2002b; Sanderson et al. 2003; Arnaud et al. 2005, and references therein). The relation derived by Arnaud et al. (2005) for a sub-sample of six relaxed clusters hotter than $3.5 \mathrm{keV}$ observed with XMM-Newton is consistent with the standard self-similar expectation, following the relation:

$h(z) M_{2500}=(1.79 \pm 0.06) \times 10^{14} M_{\odot}\left(\frac{\left\langle T_{\mathrm{X}}\right\rangle}{5 \mathrm{keV}}\right)^{1.51 \pm 0.11}$.

This result is in agreement with Chandra observations (Allen et al. 2001). In the case of RX J1347, Eq. (12) turns into an estimate of $M_{2500}=(4.07 \pm 0.46) \times 10^{14} M_{\odot}$. By considering the whole XMM-Newton sample (ten clusters in the temperature range [2-9] $\mathrm{keV}$ ), the relation steepens with a slope $\sim 1.70$
(Arnaud et al. 2005) indicating a breaking of self-similarity. In this case we estimate $M_{2500, \mathrm{DDg} 1}=(4.39 \pm 0.35) \times 10^{14} M_{\odot}$. The mass estimate that we derive at the overdensity $\Delta=2500$ differs strongly depending on the model adopted (see Table 4). From model DDg1 we estimate $M_{2500, D D g} 1=(7.10 \pm 1.40) \times 10^{14} M_{\odot}$, which is much higher than the prediction of the $M-T$ relation. The mass estimate of $M_{2500, \mathrm{NFW}}=(4.12 \pm 0.22) \times 10^{14} M_{\odot}$ as derived from the best-fitting NFW profile is instead in good agreement with the $M-T$ relation, although the large error bars prevent us from distinguishing between a self-similar or steeper relation.

\subsection{Gas mass and gas mass fraction}

From the results of the deprojected spectral analysis we compute the cumulative gas mass profile $M_{\text {gas }}(<r)$, thus obtaining values for the 6 bins used in in the spectral analysis. In order to derive better estimates when an extrapolation of the gas mass beyond $R_{\text {out }}$ is needed, we compute the gas mass profile using the radial gas density profile derived from the best fit parameters of the double $\beta$-model (model DDg1) of the surface brightness profile. The normalization of the latter is fixed using the gas density profile from the spectral analysis. The resulting gas mass profile is shown in Fig. 5. When $M_{\text {gas }}(<r)$ is evaluated within $R_{\text {out }}$ we use the binned profile and spline interpolation, which in this radial range provides values consistent with the ones computed using the results from the double $\beta$-model.

The gas mass fraction $f_{\text {gas }}$ is defined as the ratio of the total gas mass to the total gravitating mass within a fixed volume. We measure $f_{\text {gas }, 2500}=0.162 \pm 0.036$ from the mass profiles derived from the double $\beta$-model fit (model DDg1). This value is close to the global baryon fraction in the Universe, constrained by CMB observations to be $\Omega_{\mathrm{b}} / \Omega_{\mathrm{m}}=0.175 \pm 0.023$ (Readhead et al. 2004; Spergel et al. 2003), and is higher than the average value derived in a number of previous measurements with Chandra (e.g., Allen et al. 2002a; Vikhlinin et al. 2006). However, we note that a general trend of increasing $f_{\text {gas }}$ with cluster temperature (hence mass) has been observed (Vikhlinin et al. 2006). The high central gas mass fraction measured here is consistent with this tendency, as RX J1347 is a hot, massive cluster.

\section{Comparison with previous work}

In this section we compare the most relevant total and gas mass estimates for RX J1347 found in literature with our results. The values in literature are converted to the cosmology adopted here before the comparison.

\subsection{Comparison with $X$-ray studies}

Using combined ROSAT and ASCA observations Schindler et al. (1997) derived $M_{\mathrm{tot}}=1.11 \times 10^{14} M_{\odot}, M_{\mathrm{tot}}=4.93 \times$ $10^{14} M_{\odot}$, and $M_{\text {tot }}=1.45 \times 10^{15} M_{\odot}$ within $204,850,2550 \mathrm{kpc}$, respectively. These values were derived assuming isothermality 
and the error coming from the uncertainty on the global temperature is of the order of $10 \%-15 \%$, as we estimated from the plot showing the profile of the integrated total mass (see Schindler et al. 1997, Fig. 6). We find, for model (SO, DDg1), $M_{\mathrm{tot}}=$ $(1.14 \pm 0.14,0.93 \pm 0.17) \times 10^{14} M_{\odot}, M_{\text {tot }}=(8.10 \pm 0.84,7.85 \pm$ $1.60) \times 10^{14} M_{\odot}$, and $M_{\text {tot }}=(1.47 \pm 0.15,1.22 \pm 0.32) \times 10^{15} M_{\odot}$ within $204,850,2550 \mathrm{kpc}$, respectively. While we find a significant mismatch at $850 \mathrm{kpc}$, the results are in reasonably good agreement at small and large radii, in particular considering the errors and the different assumptions adopted in the mass determination. For the cumulative gas mass Schindler et al. (1997) found $M_{\text {gas }}=1.33 \times 10^{14} M_{\odot}$ and $M_{\text {gas }}=5.93 \times 10^{14} M_{\odot}$ within $850,2550 \mathrm{kpc}$ respectively, while our values are $M_{\mathrm{gas}}=$ $(1.39 \pm 0.02) \times 10^{14} M_{\odot}$ and $M_{\text {gas }}=(4.32 \pm 0.17) \times 10^{14} M_{\odot}$ within $850,2550 \mathrm{kpc}$, respectively. While the values at $850 \mathrm{kpc}$ are consistent, the large value found at 2550 by Schindler et al. (1997) is very likely due to the narrower radial range probed by their observation. As shown in Sect. 3 (see Table 1), the gas density steepens in the outer region. As a result, the gas mass derived from a single $\beta$-model fit to a narrow central region and extrapolated to large radii is biased high. In comparing the results, we should also bear in mind that the analysis presented by Schindler et al. (1997) is performed on the full $360^{\circ}$ data, as the hot enhancement in the SE quadrant has been discovered only subsequently with Chandra and XMM-Newton observations (Allen et al. 2002b; Gitti \& Schindler 2004).

We compare our best-fitting NFW profile with the one derived by Allen et al. (2002b) from Chandra data. The two profiles are consistent, with a relative difference ranging from $15 \%$ to $30 \%$ depending on the radial range considered. As a general trend, our profile results lower in the inner region (inside $\sim 600 \mathrm{kpc}$ ) and higher in the outer region (outside $\sim 1000 \mathrm{kpc}$ ) than the one derived by Allen et al. (2002b). In particular, Allen et al. (2002b) find an integrated mass within the virial radius of their best-fitting NFW mass profile of $M_{\mathrm{tot}}(<2 \mathrm{Mpc})=$ $\left(1.95_{-0.70}^{+1.48}\right) \times 10^{15} M_{\odot}$, which is in fairly good agreement with the value that we measure: $M_{\mathrm{tot}}(<2 \mathrm{Mpc})=\left(1.59_{-0.16}^{+0.18}\right) \times 10^{15} M_{\odot}$. When considering the mass profile derived from the double $\beta$ model (DDg1), which at large distances is lower than the one derived from the NFW fit (see Fig. 5 ), we find $M_{\text {tot }}(<2 \mathrm{Mpc})=$ $\left(1.11_{-0.27}^{+0.28}\right) \times 10^{15} M_{\odot}$. This value is fairly low compared to the value found by Allen et al. (2002b), but still consistent considering the errors on the mass estimates at this large distance.

Ettori et al. (2004) derive from Chandra data estimates of $M_{\mathrm{tot}}=(8.94 \pm 0.80) \times 10^{14} M_{\odot}$ and $M_{\mathrm{gas}}=(1.81 \pm 0.08) \times$ $10^{14} M_{\odot}$ within $1368 \mathrm{kpc}$, which corresponds to $r_{500}$ in their work. While our value $M_{\text {tot }}(<1368 \mathrm{kpc})=(10.84 \pm 1.11,9.72 \pm$ $2.27) \times 10^{14} M_{\odot}$ (for model SO and DDg1, respectively) agrees with the Chandra estimate, we find a larger value for the gas mass: $M_{\text {gas }}(<1368 \mathrm{kpc})=(2.35 \pm 0.05) \times 10^{14} M_{\odot}$. The discrepancy might be related to the different approaches adopted for the calculation. We estimate the gas mass directly from the density profile derived from the deprojected spectral analysis (Sect. 4.3). The gas mass computed by Ettori et al. (2004) is derived by estimating the central electron density from the combination of the best-fit results of the spectral and imaging analyses (namely the normalization of the thermal spectrum and the parameters of the single $\beta$-model). In particular, the low value measured by Ettori et al. (2004) might be biased by an underestimate of the central density due to a possible undersampling of the cluster luminosity within the radius where the thermal spectrum is extracted, which corresponds to only $\sim 0.4$ times the radius where the $\mathrm{X}$-ray emission is detectable in the Chandra data.

\subsection{Comparison with dynamical estimates}

Using the virial approach, Cohen \& Kneib (2002) derive $M_{\text {tot }}=$ $\left(6.92_{-1.89}^{+2.20}\right) \times 10^{14} M_{\odot}$ within $597 \mathrm{kpc}$ from galaxies velocity dispersion measurements. Within this radius we consistently find $M_{\mathrm{tot}}=(6.06 \pm 0.61,6.05 \pm 1.14) \times 10^{14} M_{\odot}$ for model $(\mathrm{SO}$ DDg1).

\subsection{Comparison with gravitational lensing}

Since the gravitational lensing analysis measures the projected total mass distribution, in order to compare consistently the results from the X-ray and lensing techniques we project along the line of sight the cumulative 3D mass profiles $M_{\text {tot }}(<r)$ derived in Sect. 6, thus obtaining $M_{\text {tot }}^{\text {proj }}(<r)$.

From a weak lensing investigation, Fischer \& Tyson (1997) derive $M_{\text {tot }}^{\text {proj }}=(9.35 \pm 2.55) \times 10^{14} M_{\odot}$ within $850 \mathrm{kpc}$. We find, in good agreement, $M_{\mathrm{tot}}^{\mathrm{proj}}=(9.95 \pm 1.03,9.17 \pm 2.13 .79) \times$ $10^{14} M_{\odot}$ for model (SO, DDg1). Note that Fischer \& Tyson (1997) compare their mass measurement $M_{\text {tot }}^{\text {proj }}$ within $850 \mathrm{kpc}$ with the $M_{\text {tot }}$ value quoted in Schindler et al. (1997) and find a large discrepancy. As pointed out by Sahu et al. (1998), the two mass determinations are in agreement if the correct quantities are compared.

In the strong lensing analysis by Sahu et al. (1998), $M_{\mathrm{tot}}^{\text {proj }}=$ $5.36 \times 10^{14} M_{\odot}$ is measured within $204 \mathrm{kpc}$, the cluster-centric distance of the arcs. Within this projected distance we find $M_{\mathrm{tot}}^{\mathrm{proj}}=(2.38 \pm 0.27,2.05 \pm 0.37) \times 10^{14} M_{\odot}$ for model $(\mathrm{SO}$ DDg1). Although this discrepancy might be due to the fact that we excise the perturbed region of the cluster, we note that such a large mismatch between the masses determined from X-rays and strong lensing is commonly found (see Wu et al. 1998, and references therein). In the inner core of clusters, where strong lensing occurs, the physics of the ICM may be complicated by the interaction with the central AGN. The central cluster region is thus poorly described by the usual simple models used in the X-ray methods which rely on the assumptions of spherical symmetry and hydrostatic equilibrium.

We compare our total mass determination with the lensing results of Bradač et al. (2005b). The results of Bradač et al. (2005b) are obtained using a mass reconstruction method which combines strong and weak gravitational lensing data and effectively breaks the mass-sheet degeneracy (Bradač et al. 2005a). In Fig. 6 we show the X-ray to lensing mass ratio $M_{\text {lensing }} / M_{\mathrm{X} \text {-ray }}$ as a function of radius up to $\sim 670 \mathrm{kpc}$, the limiting radius of the lensing study. From a visual inspection of this figure it is clear that there is lack of agreement between the X-ray and lensing mass estimates. Only in the central region the X-ray mass is marginally consistent with the lensing mass. The mass ratios increase with radius and tend to approach a constant value at large radii. At $600 \mathrm{kpc}$ the ratio is $2.07,2.17$, and 2.45 for the X-ray mass estimated using the single $\beta$-model (SO), double $\beta$-model (DDg1), and NFW model, respectively. We stress that the same discrepancy is found when we compare our mass profiles with a corrected mass profile computed from the lensing map where the SE quadrant, which contains the hot X-ray subclump, is excluded.

As a private communication after this work was accepted for publication, Marusa Bradac pointed out that the lensing masses reconstructed outside $\sim 510 \mathrm{kpc}$ are not very reliable because 


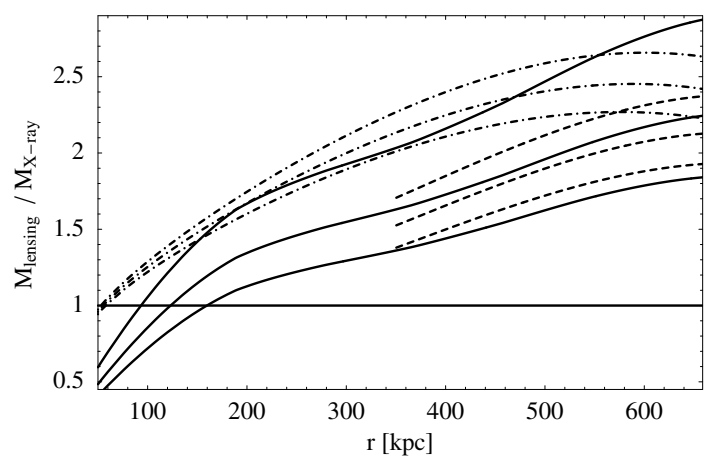

Fig. 6. Ratio of the lensing to the (projected) X-ray mass profile for different X-ray mass estimates. The line styles are the same as in Fig. 5. The reported errors are those coming from the X-ray mass determinations.

of the large error bars, and also very small radii have a significant error. This, however, does not change the main conclusion derived from the comparison with X-ray masses, since a general large discrepancy is found at all radii.

\subsection{Comparison with the Sunyaev-Zel'dovich (SZ) effect}

Through the SZ effect, Pointecouteau et al. (2001) measure the gas mass of RX J1347. They compare their results with the X-ray results of Schindler et al. (1997), finding good agreement. Within 74 arcsec $=427 \mathrm{kpc}$ the SZ estimate is $M_{\text {gas }}=$ $(4.7 \pm 0.4) \times 10^{13} M_{\odot}$ in agreement with our value, $M_{\text {gas }}=$ $(5.5 \pm 0.1) \times 10^{14} M_{\odot}$.

\section{The cool core}

As shown in Sect. 5, there is no evidence for very low temperature gas in the core of RX J1347, suggesting that the description of the inner region of this cluster by means of a standard cooling flow model is not appropriate. The spectral analysis in Gitti \& Schindler (2004) shows that if the cool core in RX J1347 is fitted with an empirical cooling flow model where the lowest temperature is left as a free parameter, very tight constraints on the existence of a minimum temperature $(\sim 2 \mathrm{keV})$ are found. This situation is common for cool core clusters and it has become clear that the gas with short cooling time at the center of these objects must be prevented from cooling below the observed central temperature minimum. The most appealing mechanism is heating by AGN because it is strongly motivated by observations. Central AGNs with strong radio activity are found in the majority of cool core clusters (e.g., Burns 1990; Ball et al. 1993) and powerful interactions of the radio sources with the ICM are observed (e.g., Bîrzan et al. 2004; Rafferty et al. 2006, and references therein). The presence of a central AGN in RX J1347 is indicated by the NRAO VLA Sky Survey (NVSS), that shows a strong central source along with some hint of a possible extended emission. However, the resolution and sensitivity of the NVSS are not sufficient to study the characteristics of the central source and establish the existence of diffuse emission. We obtained new VLA data in order to further investigate the nature and properties of the radio source in RX J1347 (Gitti et al. 2007b).

In order to explore the heating by AGN, we adopt the model developed by Ruszkowski \& Begelman (2002, hereafter effervescent heating). The details of the model and the procedure adopted to estimate the AGN parameters from the observed temperature and density profiles are given in Piffaretti \& Kaastra (2006). Here we simply summarize the essential elements. In the effervescent heating scenario the central AGN is assumed to inject buoyant bubbles into the ICM, which heat the ambient medium by doing $P \mathrm{~d} V$ work as they rise and expand adiabatically. In addition, besides being essential in stabilizing the model, thermal conduction transports energy from the hotter, outer region to the central region. Unfortunately its efficiency is poorly known since it depends on magnetic fields and models with different fractions $f_{\mathrm{c}}$ of the Spitzer rate are studied. For a fixed $f_{\mathrm{c}}$ between 0 and $1 / 3$ (the maximum for magnetized a plasma) the contribution of heat conduction as a function of radius is known since the temperature gradient is estimated from the deprojected temperature profile. We note that if one assumes that heat conduction alone balances radiative losses, then its efficiency would be much larger that $1 / 3$ of the Spitzer rate and therefore unrealistic. The raising entropy profile (in Sect. 5.3) indicates that convection is not operating on the scales that we are able to resolve and is therefore not included in the model. The extra heating profile resulting from subtracting the heat conduction yield from the ICM emissivity is then assumed to be balanced by the AGN heating function:

$H^{\mathrm{AGN}} \propto \frac{L}{4 \pi r^{2}}\left(1-\mathrm{e}^{-r / r_{0}}\right)\left(\frac{p}{p_{0}}\right)^{\left(\gamma_{\mathrm{b}}-1\right) / \gamma_{\mathrm{b}}} \frac{1}{r} \frac{\mathrm{d} \ln p}{\mathrm{~d} \ln r}$

where $p$ is the ICM pressure ( $p_{0}$ some reference value) and $\gamma_{\mathrm{b}}$ the adiabatic index of the buoyant bubbles, which is fixed to $4 / 3$ (i.e., relativistic bubbles). Fitting Eq. (13) to the extra heating profile provides the AGN parameters $L$ (the time-averaged luminosity) and $r_{0}$ (the scale radius where the bubbles start rising in the ICM). Only if $0.10 \leq f_{\mathrm{c}} \leq 0.27$ the fitting provides meaningful results. For $f_{\mathrm{c}}=0.27$ the AGN parameters are $L=7.45 \times 10^{45} \mathrm{erg} \mathrm{s}^{-1}$ and $r_{0}=4 \mathrm{kpc}$. As we decrease $f_{\mathrm{c}}$ both AGN parameters increase monotonically and reach the maximum at $f_{\mathrm{c}}=0.10$, for which we find $L=10.11 \times 10^{45} \mathrm{erg} \mathrm{s}^{-1}$ and $r_{0}=29 \mathrm{kpc}$. The trend of the AGN parameters with $f_{\mathrm{c}}$ indicates that, in the framework of the effervescent heating scenario, heat conduction and AGN heating cooperate in quenching radiative cooling. The inferred AGN time-averaged luminosity lies therefore in a quite small range $\left(7.45-10.11 \times 10^{45} \mathrm{erg} \mathrm{s}^{-1}\right)$, and is larger but comparable to the cluster luminosity in the energy range [2.0-10.0] $\mathrm{keV}\left(L_{X}=6.2 \pm 0.2 \times 10^{45} \mathrm{erg} \mathrm{s}^{-1}\right)$. The model with $f_{\mathrm{c}}=0.22$ is the one with the smallest reduced $\chi^{2}$ and in this case $L=8.32 \times 10^{45} \mathrm{erg} \mathrm{s}^{-1}$ and $r_{0}=13 \mathrm{kpc}$.

The effervescent heating model applied to RX J1347 predicts that the scale where the bubbles start rising in the ICM is in the range 4-29 kpc. The observed extension of the AGN jets should be of the same order of magnitude (Piffaretti \& Kaastra 2006). Interestingly, the first results from 1.4 GHz VLA observations of the central region of RX J1347 show hints of faint structures emanating from the discrete radio source out to $\sim 20 \mathrm{kpc}$ from the center (Gitti et al. 2007b). A comparison between the derived luminosity $L$ with the observed AGN luminosity is unfortunately not possible. In fact, in the framework of the effervescent heating model, the derived AGN luminosity is a time-averaged total AGN power and a fair comparison is possible only if the total jet power is estimated (X-ray and radio powers are known to be poor tracers of the total AGN power). At present, this was done only for M 87 in the Virgo cluster (Owen et al. 2000).

\section{Summary}

As indicated by previous studies (Allen et al. 2002b; Pointecouteau et al. 2001; Gitti \& Schindler 2004), the cluster RX J1347 shows both the signatures of strong cooling flow and subcluster merger, that are rarely observed in the same system. 
We analyze the data excluding the SE quadrant, where the presence of a hot X-ray subclump is suggesting that a minor merger has recently occurred or is still going on, and find that:

- the features (shape, normalization, scaling properties) of density, temperature, entropy, and cooling time profiles are fully consistent with those of relaxed, cool core clusters, with no indications of perturbations that may originate from the disturbed region of the cluster.

The usual assumptions of hydrostatic equilibrium and spherical symmetry can therefore be adopted when analyzing the data with the SE quadrant excluded. This allows us to perform a detailed mass reconstruction by starting from the temperature and density profiles derived from the X-ray analysis. We find that:

- the total mass profiles computed from a double and single $\beta$-model for the surface brightness give consistent results if the cool core is excised in the latter case;

- there is a reasonably good agreement between the total mass profile estimated from a double $\beta$-model and from the assumption of a NFW profile. The differences between these estimates might come from a poor spatial resolution of the density and temperature profiles in the central region, which could bias the NFW method;

- the characteristic radii $r_{\Delta}$ computed from the mass profile are in agreement with the observed size-temperature relation, although they are systematically lower than those derived by calibrating the relation with numerical simulations. The mass estimated from the NFW profile is in agreement with the observed mass-temperature relation, whereas that derived from the double $\beta$-model profile is a factor $\sim 1.7$ higher.

We compare our gas and total mass estimates with previous work and find that:

- our estimates of gas and total mass are generally in good agreement with those from previous X-ray, dynamical, weak lensing and SZ studies;

- a discrepancy of a factor $\sim 2$ between strong lensing and $\mathrm{X}$-ray mass determinations is confirmed;

- there is a large discrepancy at all radii between our total mass estimate and the mass reconstructed through the combination of both strong and weak lensing.

We study the AGN heating in RX J1347 by applying the effervescent heating model. We find support to the picture that AGN heating and heat conduction cooperate in balancing radiative losses. Our predictions concerning the extension of the AGN jets in RX J1347 are consistent with recent radio observations of the radio source at the cluster center.

Acknowledgements. This work is based on observations obtained with XMMNewton, an ESA science mission with instruments and contributions directly funded by ESA Member States and the USA (NASA). We thank the referee for insightful comments which improved the presentation of the results. We thank M. Bradač for providing the lensing results. M.G. thanks S. Ettori and F. Brighenti for useful discussions. M.G. acknowledges the support by Austrian Science Foundation FWF grant P15868, by Grant NNG056K87G from NASA's Goddard Space Flight Center and by NASA Long Term Space Astrophysics Grant NAG4-11025. RP acknowledges support from the Tiroler Wissenschaftsfond (Gefördert aus Mitteln des vom Land Tirol eingerichteten Wissenschaftsfonds). SS aknowledges support from the Austrian Science Foundation FWF under grant P18SL3-N1G.
Note added in proof. Recent (24 December 2005) publicy- available observations of the field of IGR J11305-6256, performed with the XRT instrument onboard the Swift satellite, showed that the only X-ray source detected within the ISGRI error box is positionally fully consistent with star HD 100199. The accurate Xray localization $\left(\mathrm{J} 2000 ; \mathrm{RA}=1131\right.$ 06.5, Dec $=-62^{\circ} 566^{\prime} 46^{\prime \prime} .6$, error radius: 6.') affored with XRT lies 3'.5 from HD 100199 and thus proves beyond any reasonable doubt that, indeed, this star is the optical counterpart of IGR J11305-6256.

\section{References}

Allen, S. W., Schmidt, R. W., \& Fabian, A. C. 2001, MNRAS, 328, L37 Allen, S. W., Schmidt, R. W., \& Fabian, A. C. 2002a, MNRAS, 334, L11 Allen, S. W., Schmidt, R. W., \& Fabian, A. C. 2002b, MNRAS, 335, 256 Anders, E., \& Grevesse, N. 1989, Geochim. Cosmochim. Acta, 53, 197 Arnaud, K. A. 1996, Astronomical Data Analysis Software and Systems V, ed. G. Jacoby and J. Barnes, ASP Conf. Ser., 101, 17

Arnaud, M., \& Evrard, A. E. 1999, MNRAS, 305, 631

Arnaud, M., Neumann, D. M., Aghanim, N., et al. 2001, A\&A, 365, L80

Arnaud, M., Majerowicz, S., Lumb, D., et al. 2002, A\&A, 390, 27

Arnaud, M., Pointecouteau, E., \& Pratt, G. W. 2005, A\&A, 441, 893

Ball, R., Burns, J. O., \& Loken, C. 1993, AJ, 105, 53

Bîrzan, L., Rafferty, D. A., McNamara, B. R., et al. 2004, ApJ, 607, 800

Borgani, S., Governato, F., Wadsley, J., et al. 2002, MNRAS, 336, 409

Bradač, M., Schneider, P., Lombardi, M., \& Erben, T. 2005a, A\&A, 437, 39

Bradač, M., Erben, T., Schneider, P., et al. 2005b, A\&A, 437, 49

Bryan, G. L., \& Norman, M. L. 1998, ApJ, 495, 80

Burns, J. O. 1990, AJ, 99, 14

Cavaliere, A., \& Fusco-Femiano, R. 1976, A\&A, 49, 137

Cohen, J. G., \& Kneib, J.-P. 2002, ApJ, 573, 524

De Grandi, S., \& Molendi, S. 2002, ApJ, 567, 163

Dickey, J. M., \& Lockman, F. J. 1990, ARA\&A, 28, 215

Donahue, M., Horner, D. J., Cavagnolo, K. W., \& Voit, G. M. 2006, ApJ, 643, 730

Ettori, S., Fabian, A. C., Allen, S. W., \& Johnstone, R. M. 2002a, MNRAS, 331, 635

Ettori, S., De Grandi, S., \& Molendi, S. 2002b, A\&A, 391, 841

Ettori, S., Tozzi, P., Borgani, S., \& Rosati, P. 2004, A\&A, 417, 13

Evrard, A. E., \& Henry, J. P. 1991, ApJ, 383, 95

Evrard, A. E., Metzler, C. A., \& Navarro, J. F. 1996, ApJ, 469, 494

Finoguenov, A., Reiprich, T. H., Böhringer, H. 2001, A\&A, 368, 749

Fischer, P., \& Tyson, J. A. 1997, AJ, 114, 14

Ghizzardi, S., 2001, XMM-SOC-CAL-TN-0022 at

http://xmm.vilspa.esa.es/external/xmm_sw_cal/calib/ documentation.shtml\#XRT

Gitti, M., \& Schindler, S. 2004, A\&A, 427, L9

Gitti, M., McNamara, B. R., Nulsen P. E. J., \& Wise, M. W. 2007a, ApJ, 660, 1118

Gitti, M., Ferrari, C., Domainko, W., Feretti, L., \& Schindler, S. 2007b, A\&A, 470, L25

Kaastra, J. S., Tamura, T., Peterson, J. R., et al. 2004, A\&A, 413, 415

Komatsu, E., Matsuo, H., Kitayama, T., et al. 2001, PASJ, 53, 57

Lumb, D. H., Warwick, R. S., Page, M., \& De Luca, A. 2002, A\&A, 389, 93

Macciò, A. V., Dutton, A. A., van den Bosch, F. C., et al. 2007, MNRAS, 326

McCarthy, I. G., Balogh, M. L., Babul, A., et al. 2004, ApJ, 613, 811

Markevitch, M., Forman, W. R., Sarazin, C. L., \& Vikhlinin, A. 1998, ApJ, 503, 77

Nagai, D., Vikhlinin, A., \& Kravtsov, A. V. 2007, ApJ, 655, 98

Navarro, J. F., Frenk, C. S., \& White, S. D. M. 1996, ApJ, 462, 563

Owen, F. N., Eilek, J. A., \& Kassim, N. E. 2000, ApJ, 543, 611

Piffaretti, R., \& Kaastra, J. S. 2006, A\&A, 453, 423

Piffaretti, R., Jetzer, Ph., Kaastra, J. S., \& Tamura, T. 2005, A\&A, 433, 101

Pointecouteau, E., Giard, M., Benoit, A., et al. 2001, ApJ, 552, 42

Ponman, T. J., Bourner, P. D. J., Ebeling, H., \& Böhringer, H. 1996, MNRAS, 283, 690

Ponman, T. J., Sanderson, A. J. R., \& Finoguenov, A. 2003, MNRAS, 343, 331

Pratt, G. W., \& Arnaud, M. 2005, A\&A, 429, 791

Pratt, G. W., Arnaud, M., \& Pointecouteau, E. 2006, A\&A, 446, 429

Pratt, G. W., Böhringer, H., Croston, J. H., et al. 2007, A\&A, 461, 71

Rafferty, D. A., McNamara, B. R., Nulsen, P. E. J., \& Wise, M. W. 2006, ApJ, 652,216

Rasia, E., Ettori, S., Moscardini, L., et al. 2006, MNRAS, 369, 2013

Readhead, A. C. S., Myers, S. T., Pearson, T. J., et al. 2004, Science, 306, 836 
Ruszkowski, M., \& Begelman, M. C. 2002, ApJ, 581, 223

Sahu, K. C., Shaw, R. A., Kaiser, M. E., et al. 1998, ApJ, 492, L125

Sanderson, A. J. R., Ponman, T. J., Finoguenov, A., et al. 2003, MNRAS, 340, 989

Schindler, S. 1996, A\&A, 305, 756

Schindler, S., Guzzo, L., Ebeling, H., Boehringer, H., Chincarini, G., et al. 1995, A\&A, 299, L9

Schindler, S., Hattori, M., Neumann, D. M., \& Boehringer, H. 1997, A\&A, 317 646
Spergel, D. N., Verde, L., Peiris, H. V., et al. 2003, ApJS, 148, 175 Sutherland, R. S. 1998, MNRAS, 300, 321

Tozzi, P., \& Norman, C. 2001, ApJ, 546, 63

Vikhlinin, A., Markevitch, M., Murray, S. S., et al. 2005, ApJ, 628, 655

Vikhlinin, A., Kravtsov, A., Forman, W., et al. 2006, ApJ, 640, 691

Voit, G. M. 2005, Rev. Mod. Phys., 77, 207

Wu, X.-P., Chiueh, T., Fang, L.-Z., \& Xue, Y.-J. 1998, MNRAS, 301, 861 Xue, Y.-J., \& Wu, X.-P. 2000, MNRAS, 318, 715

Zhang, Y.-Y., Finoguenov, A., Böhringer, H., et al. G. 2007, A\&A, 467, 437 